Note: This is a pre-publication final draft. For the published version, please see: Rafalow, Matthew H. (2015). "n00bs, Trolls, and Idols: Boundary-Making among Digital Youth", Technology and Youth: Growing Up in a Digital World (Sociological Studies of Children and Youth, Vol. 19), Emerald Group Publishing Limited, pp. 243-266.

\title{
n00bs, Trolls, and Idols: Boundary-Making among Digital Youth
}

\author{
Matthew H. Rafalow \\ University of California-Irvine
}

Young people are commonly thought to pursue their interests online without mind to issues of privacy, safety, or an awareness of their digital social environment. Yet, to date, few attempt to document other possibilities in practice in favor of risk-oriented research. Drawing on data collected from a one-year ethnographic study of an online gaming forum inhabited by youth and young adults, I show that participants employ discursive boundary-making strategies to cultivate a regulated social environment. By wielding emic cultural categories as they interact with their peers, the community supports new participants (n00bs), wards off harmful bullies (trolls), and upholds shared ideals (idols). This study will attempt to provide a more comprehensive understanding of how peer cultures emerge and operate online, and can do so in ways that are not necessarily risky or deviant but rather can promote civility.

More often than not, youth participation online is constructed through a lens of risk and deviance. Countless news stories and scholarly publications focus attention to widespread accounts of youth victimization online by perpetrators such as adult predators as well as peers of similar ages (ABC News 2007; Olweus 2012; Smith 2012; 2014). Although literature from the sociology of childhood provide many examples of how young people are strategic actors in their social worlds, such as through peer boundary making processes and developing status orders, rarely is this agent-centered framework applied to understand empirical examples of young people inhabiting online environments (Corsaro 1997; Crosnoe and Johnson 2011; Thorne 1993).

Contemporary work on digital youth suggests that although young people are indeed capable of transgressive behavior online (Bardone-Cone and Cass 2007; Biddle et a. 2008; Bond 2012; Mitchell and Ybarra 2007), such as through cyberbullying or interacting with dangerous strangers, youth often use the internet to simply pursue their interests and find like-minded peers, like through video game communities (Ito et al. 2009). As a result, these social environments may be less hostile than many sociological studies of youth cultures in school environments where participation can be mandatory. In this paper I examine such a possibility. Through in-depth fieldwork in a youth-created, multigenerational gaming community online, I find that participants engage in boundarymaking processes with regulatory effects. As a result, this peer culture establishes an interaction order that provides support for new community members (n00bs), wards off 
bullies (trolls), and cultivates community ideals (idols). This study thus attempts to broaden our understanding of how youth and young adults collaborate with one another online and in ways that drive social order in digital contexts.

\section{Deviance Approaches to Youth Participation Online}

A growing literature focuses attention on young people's use of the internet, a fascination due, in part, to the fact that youth are among the fastest adopters of digital technologies in the last decade (Pew 2013). Popularly termed "digital natives," or children who have grown up with technologies that permit communications online, this generation is among the most internet-connected population in the United States (Zickuhr 2011; Prensky 2001). National surveys indicate that $95 \%$ of teens and $97 \%$ of young adults are online, a figure that has been stable since 2006 (Pew 2013). Additionally, teens and young adults access the internet using a wide variety of methods, including desktop computers, mobile phone technology, and internet-connected video game systems (Nielsen 2009).

Scholarly work on the subject of digital youth often examines the potential for harm online, particularly when in the presence of adult strangers. For example, studies of risky behavior among youth online have examined its capacity for promoting self-harm (Mitchell and Ybarra 2007), anorexia (Bond 2012; Bardone-Cone and Cass 2007), and suicide (Biddle et al. 2008). Research also explores how children's access to the Internet may be related to cyberbullying (Olweus 2012; Smith 2012; 2014) and exposure to pornography (Peter and Valkenburg 2007). In particular, the topic of cyberbullying is at the forefront of the public agenda after a series of stories emerged in the media (ABC News 2007). Related studies largely position youth as victims, even when they are the perpetrators of such offenses, as cyberbullies are often targets of such aggression, as well (Tokunaga 2010). Although research in the interest of promoting safety for children is incredibly important, this work rarely takes a more agent-centered approach like that articulated by the sociology of childhood. As a result, scholars miss opportunities to identify not only risk factors of internet use but also potential avenues for youth to engage online with each other and with older adults in productive ways.

\section{Youth Culture and Social Boundaries}

In contrast to the aforementioned literature on youth risk online, the sociology of childhood and youth argues that youth are actors of their own social worlds and conceives of childhood as a socially constructed, variable, and contested category (Corsaro 1997; Fine 2004; James and Prout 1997). The "youth at risk" narrative, for example, is often bound up in a developmental construction of childhood that positions youth as victims rather than active social participants (Corsaro 1997; Lesko 2001; James and Prout 1997). Rather than see childhood only through the lens of adult-driven socialization, Corsaro (1997) suggests an Interpretive Reduction model that frames peer interaction as a site where youth actively negotiate, share, and create culture among one another. Thus, in the present study, we may expect that young people are meaningful participants of their online habitats rather than simply at risk. 
Studies of youth and youth culture in offline contexts document how youth construct and negotiate complex status orders, and engage in social stratification and socialization (Adler and Adler 1998; Crosnoe 2011; Pascoe 2007; Wilkins 2008). Of particular note, Thorne (1993) finds that elementary school children engage in boundarymaking processes to identify status differences and delineate groups based on their cultural standards. Through various types of activities, such as contests, chasing, and other rituals, children affirm gendered boundaries and promote heteronormativity within their own peer cultures. Indeed, these boundary-making processes have been widely documented in other cases, such as among teens of mixed social classes (Eckert 1989; Willis 1977), gender performances and sexual orientations (Pascoe 2007), and across racial and religious statuses (Wilkins 2008). The form that boundary making may take can vary, too. For example, Milner (2004) finds that youth employ labels such as "freak," "geek," and "cool kids" to delineate the status order at school. Although this scholarship by and large focuses on the negative consequences of boundary work, we may nonetheless expect that young people engage in similar boundary-making processes when they participate online with their peers.

\section{Digital Youth Online}

Contemporary work on digital youth suggests that although young people are frequently connected to the internet they participate online in ways that are typically an extension of youth cultural participation. Ito et al. (2009) observe that youth hang out with friends online casually not unlike their face-to-face interactions with peers. Additionally, they find that young people often use the internet to pursue their interests and find communities of other peers who share their interest when peers in their local environment are not supportive of such pursuits. Thus, young people can use the internet to connect with like-minded others in ways not limited by geographic distance. Moreover, youth opt-in to some of these peer cultural environments based on their interest rather than what has been documented in existing work that focuses on peer dynamics in mandated school settings.

Another avenue in which research on digital youth distinguishes itself from more traditional studies of youth culture is by arguing that digital youth participation online presents opportunities for intergenerational interaction. Thus, while youth can and do pursue friendships online with same-aged peers that they know from both local contexts as well as other online publics, young people have more opportunities to connect with young adults and older individuals as they pursue their interests of choice (Ito et al. 2009; Ito et al. 2013). In keeping with tenets of the sociology of childhood that position youth as active agents, these contemporary studies of digital youth position them as meaningful participants even in online communities of mixed age groups (Martin 2014; Pfister 2014).

At present, video gaming is among the most populated genres of interest pursued by youth and young adults. Recent reports suggest that $97 \%$ of youth in the United Stages ages 12 to 17 play computer, console, portable, or cell phone games, and more than half of teens play for at least an hour daily (Lenhart 2008). Additionally, $81 \%$ of young adults ages 19-29 play games (Lenhart, Jones and MacGill 2008). Research on gaming shows that online gaming communities are worthy sites for empirical investigation of how young people and young adults interact with one another. For example, education 
researchers document how when young people play massively multiplayer online games, like Lineage or World of Warcraft, these games become platforms upon which participants socialize, collaborate, and even develop multimodal skills and other valued literacies (Steinkuehler 2005; 2008; 2010). Thus, we may expect that online gaming communities where youth and young adults participate may be worthwhile sites of study for social phenomenon like peer socialization and boundary-making practices (Boellstorff 2008; Kow, Young, and Tekinbas 2014; Nardi and Harris 2006; Rafalow and Tekinbas 2014).

\section{Case and Method}

This study focuses on interactions between members of a youth-created community devoted to an interest in LittleBigPlanet $2^{1}$, a video game for the Playstation 3 , with the intent to explore whether and how youth and young adults engage in boundary-making processes among their peers online and for what purposes. In what follows, I describe the video game, its companion online community, and methods for data collection and analysis.

\section{LittleBigPlanet 2: The Game}

LittleBigPlanet 2 is a side-scrolling puzzle platform game created by Media Molecule. It was released for Playstation 3 in 2011 and it sold over 350,000 games in the first week of availability (Leack 2011). The game follows a character named Sackboy as he tries to save his home world, Craftworld, from an evil inter-dimensional vacuum cleaner called the Negativitron. The video game was marketed and designed for a wide audience across age and gender.

Among the game's key features, players have opportunities to craft levels and share them within the galaxy of Craftworld. Players can create new levels and share them in the game and in forums online, and players earn publicly visible badges for creating popular levels. Of note is that the video game was produced with mind to what Ito et al. (2013) term "openly networked design." In other words, the game provides a means players to share their pursuits within the game - such as game levels they create - with their peers in other online environments. Thus, player creations and activity are relayed between the game and online forums like Sackboy Planet.

Sackboy Planet is a website centered on an interest in the Playstation 3 game LittleBigPlanet 2. This website was selected because it is similar to other online gaming communities that have been examined in other studies in that it has a shared purpose (interest in the video game) and is a youth-inclusive population (Ito et al 2009; Ito et al. 2013; Martin 2014; Rafalow and Tekinbas 2014). Sackboy Planet is an active web forum devoted to the game, and has over 1,000 active users at any time and a membership list of

\footnotetext{
${ }^{1}$ Although people may use online platforms for many purposes, such as to extend communications with offline contacts, those interviewed all reported that they found Sackboy Planet and joined the community for the sole purpose of pursuing and sharing their interest in the LittleBigPlanet 2 game with others.
} 
nearly 23,000 users in the last six years. Although it was not possible to obtain representative samples of the Sackboy Planet participant population, the forum leader and all interviewees suggest that it serves mostly teenagers and young adults (ages 13-29) and skews male. While this case does not exclusively focus on youth, it offers a lens into how digital youth, with their young adult peers, are key contributors to a regulated online environment. This case thus stands in contrast to some deviance perspectives that suggest that young people are at risk when in the presence of adult strangers online.

\section{Method}

To evaluate the culture of the online community, including any boundary-making processes employed by its participants, data was collected through two means: observation in the forums, and interviews with community members. Fieldwork took place during a nine-month period (September 2011 to May 2012) during which the websites were visited several times a week to observe public interactions in the forums and chatrooms. The study involved 60 hours of observation by its conclusion. Although the observed activities on Sackboy Planet are public for anyone with internet access to view, I made considerable efforts to notify the forum leaders and community members by several different means. First, I contacted the creator and administrator of Sackboy Planet and shared information about the study, including the research questions and methods of data collection, for their permission to participate. Second, in consultation with this administrator, I created a public forum post to make known the study purpose and period of observation non the website. It should be noted that this is not a perfect means by which to reach all participants of a community, as it would be incredibly difficult to individually gain consent for passive observation on a platform where thousands of people interact. However, this strategy maximized community awareness of the study through the aid of the community administrator to develop a public notice of research activity and direct any participants with questions to myself and/or my institutional review board.

Observation in the forums was coupled with interviews of community members throughout the period. Because I was interested in status and boundary making dynamics in the forums, I began by interviewing the creator of Sackboy Planet (a 17-year old male) as well the moderators of the community. With the permission of the creator, I posted an open call for interviewees, noting that I was looking to speak with players both new and more experienced. Interview participants were provided a link to a study information sheet, including details about the research design and how the interview will be used, and I reviewed this sheet with them and asked for verbal consent (either by voice or chat text). In consultation with my institutional review board, participants were not asked for written consent. No personal identifiers were collected about interviewees, and signed consent forms from subjects would require them to write down their names, breaching anonymity. For participants between ages 13 and 18, I followed a modified consent protocol. For these youth, I reviewed the study information sheet and attained verbal assent from both the interviewee and their parent or guardian. Risks for this population were minimized by not collecting subject identifiers, or linking interview data to their online identities (e.g., aliases), as well as by attaining verbal and not written assent from participants. 
I conducted interviews through Skype audio calls or by Skype text chat (depending on the participant preference), and used a semi-structured interview protocol to gain an understanding of how participants conceived of their experience in the community and evaluated other participants. The interview protocol followed a narrative of participation in the forums, first asking how they found the community and what their daily habits were as participants. Later, I asked how they determined how they sorted among forum content in order to determine who to follow or what to play. As participants answered these questions, I probed for examples or stories to illustrate related boundary making processes. Interviewees were also asked structured questions about their own status characteristics, including age, race-ethnicity, gender, class, education level, and geographic region. Ultimately, I report on interviews with 19 youth and young adults, as well as 5 additional respondents who are either thirty years or older or preferred to not disclose their age (see Table 1 for more detailed sample characteristics).

\begin{tabular}{lc} 
Table 1: Interview Respondent & Characteristics \\
\hline Age & 6 \\
$13-19$ & 13 \\
$20-29$ & 2 \\
$30+$ & 3 \\
Unknown & \\
Gender & 3 \\
Female & 21 \\
Male & \\
Race-Ethnicity & 1 \\
Asian & 1 \\
Black & 3 \\
Latino/a & 16 \\
White & 3 \\
Unknown & \\
Nationality & \\
United States & 21 \\
Other & 3 \\
Total N & 24 \\
\hline
\end{tabular}

A limitation of this study is that it is not possible to know the exact demographics of participants on Sackboy Planet, including age, gender, and race-ethnicity, so that I can compare how the characteristics of this interview sample may align with statistics of overall participation. While the game is marketed towards both youth and adults, it is possible - if not likely - that the community is heterogeneous in age. Thus, this paper ultimately does not report on an essential "youth culture" in the sense that I report on data collected only from youth. For example, observed individuals in the forums could include both youth and adults. However, contemporary work on youth participation in online publics can rarely claim that they are only studying observed patterns of youth as most online publics are not youth-only, unlike peer locations in face-to-face environments like schools (Ito et al. 2009). Rather, this study shows that regulatory boundary-making 
practices exist in an online community that youth, among others, inhabit, and through interviews illustrates how youth and young adults both exert agency in this boundarymaking process. In this way, I find that existing deviance-oriented perceptions of digital youth are complicated by the fact that young people can hang out online with older peers and meaningfully participate in social regulation online.

I imported anonymized data from field notes and interviews into Dedoose, a mixed-methods data analysis software. Fieldnotes and interview data are arranged into a database within the software program, and the data analysis tool allowed me to create codes and apply them to particular sections of each note or interview document. I used "open" coding to identify broad categories that emerged (i.e., "mention of boundaries"), followed by "focused" coding to unearth more detailed categorizations of the data (i.e., "n00b," "troll," and "idol") (Charmaz 2014). This coding process allowed me to identify the most common emergent themes across the sources of data, and then categorize coded excerpts into more specific typologies that are discussed in the results.

\section{WELCOME TO SACKBOY PLANET}

In the sections that follow, I illustrate the results from a grounded approach to documenting and analyzing boundary-making processes that exist within this community online. This exploratory research illuminates my process of discovery as I interfaced findings with existing research.

\section{The Role of Community Policing}

What informs relations on Sackboy Planet and among its membership, and how is interaction regulated? In order to fully participate, visitors must register and create an account on the website. Registration only requires that participants create an alias, or username, and password, but it also importantly provides the first glimpse into regulatory expectations to every community participant. The leadership of the website present a number of rules upon registration with the community. After registering an account, users are re-directed to a website e-mail dashboard that includes a welcome message automatically generated by the forum system. The message informs users that they need to read the "Rules \& Regulations" post on the website before participating in the community. Upon clicking the attached link, users find that the post elaborates on two forms of stated regulation: human moderators, and non-human, systematic regulation via the forum design. The post states that moderators, who are officials of the forums, police interaction and watch out for cheating the reputation system. The second section of the rules and regulations describes the formal reputation and infraction system in more depth:

Any time you break a rule, you will get a private message (called an 'infraction')... Once you reach a predefined number of points, the system (read: not a human being) will automatically ban you for a predefined number of days... Overall, this system will be much more standard and automated, so you can keep track of how close you are to a ban. 
Immediately upon registration with the community participants are notified of policies aimed to ward off bad behavior, which stands in contrast to existing research that positions youth engagement online as constantly fraught with risk. This rules and regulation page indicates that the primary forms of social control are through designated authorities (moderators) and more systematic reputation and infraction systems established through the forum design code.

Subsequent interviews with moderators of the forum, however, revealed that the sources and standards of the community etiquette are much more complex. Moderators do assign infractions when they witness bad behavior, but they are not the first line of defense. One moderator, ModBob (18 years old), explained: "If [community members] are doing something wrong and someone takes offense to it generally the person will let us know. Otherwise we really can't tell unless someone reports it." When describing their role, moderators regularly discussed how their policing is usually secondary to other forms of community regulation. Another moderator, Josephina (19 years old), elaborated: "Moderators just keep order and assist the new people as they come in and make them feel welcome...everyone pretty much polices themselves, helps each other out."

Moderators on Sackboy Planet viewed their role as important to the overall function of the community by assigning infractions and serving as greeters to new members, but because there are many users and so much activity in the forums they actively rely on members to report bad behavior and maintain community standards. As other work has suggested, the environment for interaction online is, in fact, different than many face-toface environments that youth inhabit because online forums can be host to potentially thousands of other anonymous or pseudononymous others. Yet, these moderators describe community regulation as one means to address this structural difference.

When I asked non-moderator community members about community regulation, they confirmed the prevalence and importance of community policing. I asked if nonmoderators ever step in and do something when they see bad behavior, and Turtle (14 years old) wholeheartedly agreed: "Plenty of times. I've done it once or twice too. I also report a lot of spam and advertising. So yea all of Sackboy Planet try to make it a nice place to be." Another member, Ponytale, responded to the same question by explaining his own self-professed role on the site as an unofficial community regulator: I've never been a moderator or administrator or anything and I don't really care to. But I'm kind of like a non-moderator moderator. I tell people what I think or diffuse situations. Or if I don't do it publicly I'll write them a private message." For these participants, community policing - not moderator-driven regulation - was an important component of maintaining collective standards and is a shared responsibility. Contrary to the framing of literature on digital youth and risk, young people can be active agents in managing behavior online and building a community regulated around a shared purpose of kindness.

\section{How Community Policing Occurs in Practice}

How does social regulation occur on Sackboy Planet, and what does community policing look like in practice? As noted in the "Rules and Regulations" post described earlier, Sackboy Planet's most prominent features include an algorithmically developed community management system. This system includes reputation markers such as various experience bars, stars, and badges on their profiles obtained through the accumulation of 
different social metrics, such as how many times they log in or how many other players like or thank their posts. In an interview with the forum leader (17 years old) and designer of the social metrics, he explains the role of these varies status indicators in shaping forum interactions:

In terms of rewards, we have the experience system...if they use the site and interact with people and help people they will earn experience for it. By posting to the site and posting blogs, by replying and making new threads users will gain experience...I guess we're printing our own kind of money.

The community leader's design of the website created a type of market based on interaction in the forums. While the leader himself does not see it as a "real money" currency, he portrayed it as a kind of market that relies primarily on social capital: being an active member of the community social network, creating discussions with other members, and replying to others' posts.

After observing in the forums for several months, I myself began to accrue trophies, or awards, that signaled my participation (see Figure 2). One award was for being registered with the community for three months. I received a message notification alerting me to my award, and the forum system automatically inserted a small picture of a trophy with the number 3 on my profile page. Other, more senior members, had much more diverse symbols of achievement on their profiles. Some of them include titles such as "Veteran" to refer to the user's extensive participation over a long period of time. Others accumulate experience across one of several bars that increase or decrease depending on the behavior of the member in the forum. Lucidio, for example, had a full orange bar to reflect that many people "liked" or thanked his comments on the blog. He had a low green bar, however, which indicated that he was a relatively infrequent participant on the forums. The different types of trophies, or badges, and reputation bars players accumulate might function as a type of status currency within the forums.

In order to interrogate the collective meaning behind these awards, I asked every interviewee to describe the various reputation bars and points that appear on forum members' publicly visible account profiles. Some said that the game metrics matter: "They do care...if your profile has 2,000 hearts then everybody knows you." Respondents described others as completely unimportant: "I personally never even look at that stuff...I don't really notice it, basically." When they expressed such indifference to the reputation system, I followed up by asking what then actually determines who is considered good or bad. Community members like Poseidon (14 years old) revealed that other status indicators inform reputation:

High reputation bars just mean that they participate a LOT in the forums. I suppose if someone is a higher level, they'd be less likely to have their posts skimmed. But if it appears to be a n00b then their post will most likely be skimmed/skipped. After a while, you really learn who the big names are on the site. :P

While it is clear that participants in this forum are active agents in creating and reproducing forms of social control on Sackboy Planet, how it is that they do so is rather 
complex. Although the stated rules and regulations clearly delineated certain expectations of the forum, moderators rely on the community to maintain its standards. Moreover, these participants see that enforcing community etiquette is a responsibility and an important way to maintain the collective good. Further interrogation of the community management system revealed that while these formal metrics do in some ways matter in shaping who is good or bad, such as when a user's level has earned thousands of hearts or plays from other players, others do not. This last quote suggests that other status indicators, such as n00b, may more meaningfully inform interaction in the community.

\section{n00bs: New and Inexperienced Participants}

Observation in the forums indicated that socialization into the appropriate standards of behavior is key not only to avoid having their behavior corrected by other participants. The following is an excerpt from a forum post:

Bluebell: "Hey guys im new to all of this, but if you could just spend 30 seconds of your time to click this link and subscribe to me, it means a lot."

Rangerguy: "Hi and welcome to Sackboy Planet! It's great to have you here, but here is a suggestion. Before asking people to subscribe to your youtube channel and play your level, maybe tell a tiny bit about yourself. What brought you to the site, your experience, etc. Most people here are nice and more than happy to help out. Look forward to seeing you around!"

Flowerpower: "Welcome to Sackboy Planet. Lots of good information above. I agree with Rangerguy, let people get to know you first."

In the above example, requesting that others' view your content before following other rules, such as sharing more about yourself and letting others get to know you first instead of making anonymous requests, results in getting ignored. Knowledge of the social rules or community etiquette represents a type of cultural knowledge that must be attained and demonstrated to attain important resources from other members, such as guidance or attention to one's shared content in the forums.

A very frequent subject of discussion by community members from both forum activity as well as interviews was the "n00b." Upon further questioning about the n00b category, I learned from community members that n00bs are identified through discourse in the forums, and come to represent people and behaviors that signal being new to the community and unsocialized into community etiquette. Turtle (14 years old) explained:

Interviewer: "How do you know someone is a n00b?"

Turtle: "If they're like ZOMGZ PLAY MY LEVELZ PLZ! YOU WILL LOVE IT! OMG! :D”

Interviewer: "hahahaha. So all caps probably!"

Turtle: "Or all lower case :D"

Interviewer: "ah, like me :)"

Turtle: "run on sentences :P" 
Turtle: ":D”

On Sackboy Planet, n00bs are the least reputable members of the community and embody numerous statuses at once. As in the above interview excerpt, n00bs use standards of grammar that are not proper English as understood by the community. n00bs use all capital lettering, run on sentences, or, as I mistakenly did, all lower case lettering. Posts from community members suggest that the n00b label also referred to a relative lack of experience. One member created a post titled, "Alert: n00b!" She then noted that she was "fairly new to the game and just wanted to say hello," noting that she "would love to receive advice on how to improve at the game." In another post, a user expressed that he is a n00b and others responded by offering guidance and support:

Banjo Bill: "Hi everyone! I'm a n00b!"

Sassypants: "Hi! Welcome n00b! You could actually probably still say I'm a n00b myself, but I'm pretty active here."

Basketbal199: "Welcome to the community, n00b. ;) We know you are new to this place, so if you want to know more about the rules there's a section you can check out on it. If you have any other questions, feel free to let me know!"

Community members frequently described n00bs as inexperienced members, and in public posts they typically offer to help professed n00bs noting that they themselves were once n00bs or inexperienced members when they first joined. In this way, these participants engage in boundary-making processes as they wield the n00b label sometimes in a jocular tone - to separate people who seem inexperienced and are in need of help to be socialized into community standards.

\section{Trolls: Intentionally Harmful Users}

Another cultural category that surfaced during observation and interviews was the "troll." On Sackboy Planet, trolls represent people who intentionally breach community etiquette and are perceived as harming other users. In one thread, members talked about a user who was trying take away the attention of people from Sackboy Planet and direct them to another website:

Frog51: "The troll who claimed to be a community member is some idiot who is swearing over and over and telling people to go to a different forum."

Goalie1991: "He's now posting this comment in every part of the forum. Someone please stop him."

BigReader: "He's trying to steal people here to go to other places, and his actions are very irresponsible and he is spamming everyone. This is unforgivable behavior!" 
In this example, swearing and drawing attention away from the forums was identified as a flagrant violation of community standards. In another post, a member complained about unkind reviews he had received on a game he created and shared with the community:

An evil troll gave my shared game a bad review. I worked really hard on it, and I got really offensive feedback. My friends remind me that not everyone will appreciate stuff you share with the community, but this troll said mean things on purpose.

In contrast to the n00b category, which described users who may be inexperienced or immature, trolls exhibited behaviors that break the rules of the community intentionally. While giving someone a bad review in another community may be seen as acceptable behavior, giving a bad review in this way on Sackboy Planet is seen as an intentional breach of etiquette and justified, for this user, calling them a troll. Behaviors that are identified as troll-like have consequences for their engagement on Sackboy Planet. In another post, community members discussed what to do when one encounters a troll:

Bluebird: "Has anyone ever had to deal with a troll? I'm having trouble because this dude is trolling me...what should I do?

Smackattack: "Ignore them."

Starfighter: "Report them to the moderators."

Rainbowbrite: "Don't feed the trolls. They don't deserve the attention."

Members of Sackboy Planet report that attention from other members is a scarce resource. In the above example, the most common response to others' troll displays is simply not responding at all. In other cases, it involves reporting them to moderators who can take more decisive action, such giving them a violation or removing them from the community. In an interview, a community member named Ninjadude (16 years old) described to me an experience when others labeled him a troll:

I called [other forum members] n00bs and all these people were sort of like in response they were making jokes and making fun of me...I edited my post to get rid of it. Basically what I'm saying is people won't be mean but they'll be sarcastic or make fun of it and it makes you stop...like this is basically what happens if you're like a troll or being mean on the forums.

For this member, both the n00b and troll status categories are enacted through discourse in the community: the community demarcates particular interactions as associated with one of the cultural categories. He described his behavior, which was interpreted as violating community standards of decency and subsequently heavily policed, as troll-like behavior. Being labeled a troll results in community retaliation in ways that correct or modify the behavior of the identified user. Like the n00b category, this user never fully identified himself as a troll. This participant's experience reflects the moderating power that discursive boundary-making has on users' future behaviors. Such status categories in this community are transient, and are primarily used through discourse to police behavior. 
While trolls and n00bs are similar categories in that they both can be used to moderate behavior online, n00bs represented players who are new or in the process of being socialized, whereas trolls are assumed to have intentionally violated the cultural etiquette. Participants use these labels to engage in boundary-making among participants in their online community, delineating types of behavior that do not meet the cultural etiquette or represent the cultural competencies valued by the community. On Sackboy Planet, categories such as n00b and troll are labels that are used strategically to uphold community standards. Cultural etiquette on the forums informs user reputation and whose content is viewed and evaluated in an attention-scarce environment.

\section{Idols: Revered Participants}

While n00bs and trolls are two emic identity categories used to identify bad behavior and socialize members into expected cultural competencies, how does the community identify ideal cultural competencies or those who display such competencies? By interrogating the last category employed in the forums - idols - I show how some users are identified and celebrated.

On Sackboy Planet, idols are celebrities in the community that often are depicted as role models. In a post titled, "Who is your idol?" community members shared people they looked up to, and why:

MisterSack: "If I had to say anyone was my idol it would be the creators of this game. They are very creative. And also the leader of this community!

Lipglossgurl: "My idol makes the most amazing game levels and has such a distinct style, always innovative!"

In another post, Raindrop1985 explained why she joined the site to begin with: "Hi everyone! I couldn't resist joining this site anymore because of Johnjohnn. He's my idol and makes the best game levels!" Another user, TennisFan, described his community idol: "She's my idol because she's a super nice person and because she makes wonderful levels. She's my favorite creator on Sackboy Planet. The designs are really nice." Community members describe idols as people connected to the Sackboy Planet community who demonstrate styles or competencies valued by the community. One important competency with the game is producing and sharing game content that the community believes reflects great skill.

In both the forums and interviews community members would regularly refer to Johnjohnn (25 years old), a user who they held in high esteem. In an interview with Johnjohnn, he explained that some time ago he had an idol in the community before he achieved celebrity himself:
JohnJohnn:
"DarkMatter9 was my idol."
Interviewer: "Why was DarkMatter9 your idol?"
JohnJohnn: "The levels he made were so awesome I wanted to know his secret. What was he able to do that I wasn't?"
Interviewer: "Was he a celebrity? Was he hard to pin down?" 
JohnJohnn: "Yes. He was kind of a celebrity...I met him through a project and we became friends."

Johnjohnn used the idol category to describe someone who was inspirational because of his skill level with game design: he had a "secret," that is, a set of cultural competencies or skills, that Johnjohnn did not know but wanted to learn. Elsewhere, Johnjohnn described DarkMatter as "inspirational," "a huge motivator" as he sought out to improve himself as a designer. Although idols can take multiple forms, such as those who find undiscovered levels and give recommendations to the community, members regularly described idols as people who became famous because of their created levels and who have the highest level of skill with game design. Much like the literature on peer cultures, these participants also engage in boundary-making practices to identify and celebrate high status members that represent their cultural ideals.

The idol status grants benefits to those who inhabit it, including access to other high status community members. DarkMatter9 (21 years old) explains:

\section{DarkMatter9: "Celebrity status helped me reach more people and I could find those people I work well with." \\ Interviewer: "So you could be choosier." \\ DarkMatter9: "Yes...and the people I work with tend to be celebrities themselves...I've never had someone successfully solicit [level design] help from me through the fan sites. I get a lot of messages from the game networks like 'Come help me fix this'... it became tiresome to be constantly helping."}

In the above example, this idol describes how he only works with or helps people who are also idols. When asked why he does not regularly help the community anymore, he expressed that he had already created extensive YouTube tutorials that can provide direction to regular members. Thus, idol status provides rewards in the form of access to other community elites, but in this community it is achieved through meeting the community expectation of helping and producing valued resources, like tutorials, for others.

Members of Sackboy Planet provide many opportunities for participants to work towards becoming an idol. Contests are very exciting and emotional ritual in the community. For example, a community leader created a challenge contest to design "the most beautiful game level that takes place entirely underwater." In his announcement post, he explained: "Hi everyone! This time around the great Ocean gave us ideas for the contest. Are you ready to take the dive?" In the subsequent paragraphs, he explained that the challenge was to design "the most creative and fun fast-paced underwater game." In addition, like other contests, he stated how it would be evaluated: through quality of "Gameplay, Originality, Aesthetic, and Sound." Community members were allotted a set amount of time to create and submit levels, and eventually the contest garnered scores of submissions that were shared publicly. All players could submit their own designed levels to be considered for awards and commendation. Contests were run by idols, and users post levels in a thread designated for the contest. These threads are highly active, and once contest participants submit their level many other players will also play them 
and leave comments that are a mix of support and critique. In the case of the water level, some players left comments saying "Great job!" or "I can't wait to play these levels!" Others were more critical: "Submissions this time around weren't that great. :- $\mid$ " or "I didn't really like X's level." Participation in community contests is an opportunity for players to display their skills publicly and receive feedback, good or bad, from other membership. This feedback is a form of participant-driven boundary making in action for idol status.

Contest rituals provide an example of how the idol category is a boundary-making process organized by the members of this community. Participants assess worthy and unworthy performances of cultural competencies, and reward those who display such competencies by spotlighting that member to the community. Although the idol label occupies less of a staple in daily forum interactions between members of Sackboy Planet, it certainly is prevalent during interviews and associated contests draw the highest views from participants than any other discussion topic. Through these practices, community leaders are able to separate the community members into low and high status groups, distinguish the idols through peer and judge evaluation, and determine whose shared content receives the most and the least attention.

\section{CONCLUSION}

Through a discussion of "n00b," "troll," and "idol," emic cultural categories to an online marketplace and community of gamers, I show youth participation online can be more complicated than existing literature suggests. Previous work that employs a deviance or risk-centered orientation to the online activities young people pursue online largely ignores that youth, with their young adult peers, are active agents in their own pursuits on the internet. Similar to existing studies from the sociology of childhood on peer cultures, the youth and young adults in this online community engage in boundarymaking processes that organize people based on their behavior in the interest of maintaining a civil community. This study illustrates how youth and young adults use labels discursively to help people in need, ward off bullies, and celebrate their ideals. 


\begin{tabular}{|c|c|c|}
\hline & $\begin{array}{l}\text { Perceived characteristics of } \\
\text { cultural category }\end{array}$ & $\begin{array}{l}\text { Effect of category on } \\
\text { community behavior }\end{array}$ \\
\hline n00b & $\begin{array}{l}\text { Inexperienced } \\
\text { Poor grammar } \\
\text { New }\end{array}$ & $\begin{array}{l}\text { Mocked for poor grammar, } \\
\text { offered help to be socialized into } \\
\text { community etiquette }\end{array}$ \\
\hline Troll & $\begin{array}{l}\text { "Idiot" } \\
\text { Swears/uses profanity } \\
\text { Irresponsible } \\
\text { Intentionally causes harm }\end{array}$ & $\begin{array}{l}\text { Participant comments are } \\
\text { skipped/skimmed, user is } \\
\text { sanctioned and/or evicted }\end{array}$ \\
\hline Idol & $\begin{array}{l}\text { Leader } \\
\text { Creative } \\
\text { Role Model } \\
\text { Innovative } \\
\text { Celebrity }\end{array}$ & $\begin{array}{l}\text { Participant is celebrated and } \\
\text { receives high attention }\end{array}$ \\
\hline
\end{tabular}

Table 2: Perceived Characteristics of Categories and Effect on Community Behavior

The cultural labels that youth and young adults employ have meaningful effects on the behavior of the online community towards its participants. As shown in Table 2, "n00b" and "troll" are emic labels used through discourse to identify inexperienced players and players who exhibit bad behavior. n00bs are described by community members as new and inexperienced, and have poor grammar. Often, identified n00bs are provided with guidance as to how to better interact in their future communications on the forums and with others on Sackboy Planet. Community members who exhibit n00b-like behavior are rarely given the attention needed to their products, and thus their formal metrics (including ratings and plays/purchases) are relatively absent any data.

"Trolls" are described by community members as "idiots," people who swear or use profanity, irresponsible, and people who intentionally cause harm to others. Content shared in the marketplace by both n00bs and trolls are typically ignored, though the reasons for ignoring are different. n00bs are believed to be new and inexperienced players who often need guidance to be socialized into the expectations of the community. Trolls, however, are thought to have intentionally violated the norms of the community. Trolls are generally handled with much more strict sanctions, including widespread attacks by other members or being kicked out of the community.

"Idol" is a cultural category used to identify players who are celebrated members and skilled level designers. They are described by the community as leaders, creative types, role models, and innovative. They are widely assumed to be celebrities on Sackboy Planet. Their creations or products are often held in high regard, and receive much attention from members in an otherwise attention scarce environment. Formal metrics for these members are at the highest levels of any user. They are regularly at the top of all the highly rated and purchased charts and their work is widely known in the community. Although the idol label is not used as regularly during forum interactions as is n00b or troll, it is a significant to the interviewed participants. Moreover, its use in the forums 
during related rituals, like contests, attain the highest views of any discussion topic in the forums. Idols come to represent the ideals of those who inhabit Sackboy Planet.

This study has a number of limitations that merit further investigation in future work. I was not able to obtain demographic data for the Sackboy Planet community as a whole, which makes it difficult to know the ages of those interacting in the public forums. It is also not possible to determine if the interview sample represents a demographic portrait of the community as a whole. However, despite these limitations, interviews with the forum leader, as well as other participants, suggests that the community includes youth and young adults and is multigenerational. Thus, the findings illustrated in this paper contest deviance-oriented perspectives on youth participation online by showing that they are meaningful participants in online social regulation - even in the presence of older peers. Yet, a study of an online community where demographic data is known would provide a clearer portrait the experience, and it would provide more substantial evidence that youth are powerful forces as compared with other membership in the community.

This work suggests that research on young people's participation online need be cognizant of the capacity for youth to engage in not only risky activities but also be active agents in the development of a space for civil and safe interactions with their peers. As compared with other deviance-oriented studies that paint youth participation online as associated with cyberbullying, anorexia, bulimia, self-harm, and pornography (BardoneCone and Cass 2007; Biddle et a. 2008; Bond 2012; Mitchell and Ybarra 2007), I find that young people and their older peers collaboratively produce a regulated environment. Indeed, the challenges that youth face to manage their community are sometimes different online than has been identified by literature on peer cultures in schools (Adler and Adler 1998; Crosnoe 2011; Eckert 1989; Pascoe 2007; Thorne 1993; Wilkins 2008; Willis 1977). As described by the youth moderators of the forums, there are sometimes thousands of people interacting with each other in their community at any given time. Thus, appointed moderators are not able to observe every interaction. The youth in this community employ crowd management boundary-making strategies that rely on the broad populace to participate and help make it, in the words of one youth, "a nice place to be." 


\section{References}

ABC News. (2007). Parents: Cyber bullying led to teen's suicide. $<$ http:// abcnews.go.com/GMA/story?id=3882520\&page=1 $>$ Retrieved 20.01.10.

Adler, P.A., and Adler, P. (1998) Peer power. Rutgers University Press.

Biddle, L., Donovan, J., Hawton, K., Kapur, N., \& Gunnell, D. (2008). Suicide and the internet. British Medical Journal, 336, 800-802.

Bardone-Cone, A.M., and Cass, K.M. (2007). What does viewing a pro-anorexia website do? An experimental examination of website exposure and moderating effects. International Journal of Eating Disorders 40(6): 537-438.

Boellstorff, T. (2008) Coming of age in Second Life: An anthropologist explores the virtually human. Princeton: Princeton University Press.

Bond, E. (2012). Virtually Anorexic - Where's the harm? A research study on the risks of pro-anorexia websites. Available from:

http://www.ucs.ac.uk/SchoolsAndNetwork/UCSSchools/SchoolofAppliedSocialS ciences/Virtually\%20 Anorexic.pdf [last accessed 19 December 2013].

Charmaz, K. (2014). Constructing grounded theory. London: Sage.

Corsaro, W.A. (1997). The Sociology of Childhood. Thousand Oaks, CA: Sage Publications, Inc.

Crosnoe, R. (2011). Fitting in, standing out: Navigating the social challenges of high school to get an education. Cambridge: Cambridge University Press.

Crosnoe, R. and Johnson, M.K. (2011). "Research on Adolescence in the $21^{\text {st }}$ Century." Annual Review of Sociology 37: 439-460.

Eckert, P. (1989). Jocks and Burnouts: Social Categories and Identity in the High School. New York: Teachers College Press.

Fine, G.A. (2004). Adolescence as Cultural Toolkit: High School Debate and the Repertoires of Childhood and Adulthood. The Sociological Quarterly 45(1): 120.

Ito, M. et al. (2009). Hanging Out, Messing Around, and Geeking Out: Kids Living and Learning with New Media. Cambridge, MA: MIT Press.

Ito, M., Gutiérrez, K., Livingstone, S., Penuel, B., Rhodes, J., Salen, K., Schor, J., SeftonGreen, J., and Watkins S.C. (2013). Connected Learning: An Agenda for Research and Design. Irvine, CA: Digital Media and Learning Research Hub.

James, A, Jenks, C., and Prout, A. (1998). Theorizing Childhood. Cambridge: Polity Press.

Kow, Y.M., Young, T. and Tekinbaş, K.S. (2014). Crafting the Metagame: Connected Learning in the StarCraft II Community. Irvine, CA: Digital Media and Learning Research Hub.

Leack, J. (2011). January 2011 NPD Results Show Some Software Surprises. PlayStation LifeStyle, 18 Jan, http://playstationlifestyle.net/2011/02/18/january2011-npd-results-show-some-software-surprises/, accessed 29 January 2013.

Lenhart, A. (2008). “Teens, Video Games and Civics." Washington, DC: Pew Internet \& American Life Project.

Lenhart, A., Jones, S., and MacGill, A. (2008). "Adults and Video Games." Washington, DC: Pew Internet \& American Life Project.

Lesko, N. (2001). Act Your Age!: A Cultural Construction of Adolescence. New York: 
RoutledgeFalmer.

Martin, C. 2014. Learning the Ropes: Connected Learning in a WWE Fan

Community. Irvine, CA: Digital Media and Learning Research Hub.

Mitchell, K.J., \& Ybarra, M.L. (2007). Online behavior of youth who engage in self-harm provides clues for preventive intervention. Preventive Medicine, 45, 392-396.

Nardi, B., \& Harris, J. (2006). Strangers and Friends: Collaborative Play in World of Warcraft. Proc. CSCW '06, 149-158.

The Nielsen Company. (2009). Youth and media...Television and beyond. New York: The Nielsen Company.

Olweus, D. (2012). Cyberbullying: An overrated phenomenon? European Journal of Developmental Psychology, 9, 520-538.

Pascoe, C. J. (2007). Dude, You're a Fag: Masculinity and Sexuality in High School. University of California Press.

Peter, J., \& Valkenburg, P. (2011). Adolescents' online privacy: Toward a developmental perspective. In S. Trepte \& L. Reinecke (Eds.), Privacy online (pp. 221-234). Heidelberg: Springer.

Pfister, R.C. 2014. Hats for House Elves: Connected Learning and Civic Engagement in Hogwarts at Ravelry. Irvine, CA: Digital Media and Learning Research Hub.

Maddem, M., Lenhart, A., Duggan, M., Cortseni, S. and Gasser U. (2013). Teens and Technology 2013. Washington, DC: Pew Internet \& American Life Project.

Martin, C. (2014). Learning the Ropes: Connected Learning in a WWE Fan Community. Irvine, CA: Digital Media and Learning Research Hub.

Prensky, M. (2001). Digital natives, digital immigrants. On the Horizon, 9, 1-2.

Rafalow, M.H., and Tekinbaş, K.S. (2014). Welcome to Sackboy Planet: Connected Learning Among LittleBigPlanet 2. Irvine, CA: Digital Media and Learning Research Hub.

Smith, P.K. (2012). Cyberbullying and cyber aggression. In S.R. Jimerson, A.B. Nickerson, M.J. Mayer \& M.J. Furlong (Eds.), Handbook of school violence and school safety: International research and practice, 2nd ed. (pp. 93-103). New York: Routledge.

Smith, P.K. (2014). Understanding school bullying: Its nature and prevention strategies. London: Sage.

Steinkuehler, C.A. (2005). "The New Third Place: Massively Multiplayer Online Gaming in American Youth Culture." Journal for Teacher Education (special issue, edited by Gun-Marie Frånberg).

Steinkuehler, C.A. (2008). "Massively multiplayer online games as an educational technology: An outline for research." Educational Technology, 48(1), 10-21.

Steinkuehler, C.A. (2010). "Video games and digital literacies." Journal of Adolescent \& Adult Literacy, 54(1), 61-63.

Thorne, B. (1993). Gender Play: Girls and Boys in School. New Brunswick, NJ: Rutgers University Press.

Tokunaga, R.S. (2010). Following you home from school: A critical review and synthesis of research on cyberbullying victimization. Computers in Human Behavior, 26, 277-287.

Willis, P.E. (1977) Learning to labor: How working class kids get working class jobs. 
New York: Columbia University Press.

Wilkins, A.C. (2008) Wannabes, goths, and christians: The boundaries of sex, style, and status. Chicago: University of California Press.

Zickuhr, K. "Generations and their gadgets." Washington, DC: Pew Internet \& American Life Project. 